\title{
A nomogram to predict overall survival of patients with early stage non-small cell lung cancer
}

\author{
Jiahui Zhang ${ }^{1 \#}$, Jingyi Fan ${ }^{1 \#}$, Rong Yin ${ }^{2,3 \#}$, Liguo Geng ${ }^{1}$, Meng Zhu ${ }^{1,3}$, Wei Shen ${ }^{1}$, Yuzhuo Wang ${ }^{1}$, \\ Yang Cheng ${ }^{1}$, Zhihua $\mathrm{Li}^{1}$, Juncheng Dai ${ }^{1,3}$, Guangfu Jin ${ }^{1,3}$, Zhibin $\mathrm{Hu}^{1,3}$, Hongxia $\mathrm{Ma}^{1,3}$, Lin $\mathrm{Xu}^{2,3}$, \\ Hongbing Shen ${ }^{1,3}$
}

${ }^{1}$ Department of Epidemiology, Center for Global Health, School of Public Health, Nanjing Medical University, Nanjing 211166, China; ${ }^{2}$ Department of Thoracic Surgery, Nanjing Medical University Affiliated Cancer Hospital, Jiangsu Key Laboratory of Molecular and Translational Cancer Research, Cancer Institute of Jiangsu Province, Nanjing 210009, China; '3iangsu Key Lab of Cancer Biomarkers, Prevention and Treatment, Collaborative Innovation Center for Cancer Medicine, Nanjing Medical University, Nanjing 211166, China

Contributions: (I) Conception and design: H Shen; (II) Administrative support: H Shen; (III) Provision of study materials or patients: W Shen, Y Wang, Y Cheng, Z Li, G Jin, Z Hu, H Ma, L Xu; (IV) Collection and assembly of data: J Zhang, J Fan, R Yin, L Geng, M Zhu, J Dai; (V) Data analysis and interpretation: J Zhang, J Fan, R Yin, L Geng; (VI) Manuscript writing: All authors; (VII) Final approval of manuscript: All authors.

"These authors contributed equally to this work.

Correspondence to: Hongxia Ma. Department of Epidemiology, Center for Global Health, School of Public Health, Nanjing Medical University, 101 Longmian Rd., Nanjing 211166, China. Email: hongxiama@njmu.edu.cn. Lin Xu. Department of Thoracic Surgery, Nanjing Medical University Affiliated Cancer Hospital, 42 Baiziting, Nanjing 210009, China. Email: xulin83@vip.sina.com; Hongbing Shen. Department of Epidemiology, Center for Global Health, School of Public Health, Nanjing Medical University, 101 Longmian Rd., Nanjing 211166, China.

Email: hbshen@njmu.edu.cn.

Background: Nomograms have been widely used for estimating cancer prognosis. The aim of this study was to construct a clinical nomogram that would well predict overall survival of early stage non-small cell lung cancer (NSCLC) patients after surgery resection.

Methods: A total of 443 patients diagnosed with pathologic stage I and II NSCLC who had undergone curative resection without neoadjuvant chemotherapy or radiotherapy were recruited and analyzed. The logrank test and multivariate Cox regression analysis were used to select the most significant predictors in the final nomogram for predicting overall survival. Furthermore, the model was validated by bootstrap methods and measured by concordance index (C-index) and calibration plots.

Results: Four independent predictors for overall survival were identified and included into the delineation of the nomogram (tumor differentiation, station of sampled lymph nodes, pathologic $\mathrm{T}$ and pathologic $\mathrm{N}$ ). The model showed comparatively stable discrimination (bootstrap-corrected C-index $=0.622,95 \% \mathrm{CI}$ : 0.572-0.672) and good calibration.

Conclusions: We successfully developed a nomogram incorporating available clinicopathological variables to predict overall survival of early stage NSCLC patients after surgery resection, which might help clinician select better appropriate treatment decisions.

Keywords: Lung cancer; nomogram; survival; TNM stage; stage I and II

Submitted Mar 21, 2018. Accepted for publication Oct 31, 2019.

doi: $10.21037 /$ jtd.2019.11.53

View this article at: http://dx.doi.org/10.21037/jtd.2019.11.53 


\section{Introduction}

Lung cancer remains the most common cause of cancer death worldwide (1), with non-small cell lung cancer (NSCLC) accounting for the major histology (2). For early stage NSCLC, surgery resection is the best treatment, with 5 -year overall survival of only $36-73 \%$ (3). The TNM staging system based on the extent of the primary tumor, involvement of regional lymph nodes and spread to distant sites, is widely used as a guide for predicting prognosis of lung cancer in clinical practice (3). Moreover, the wide range of survival rates in NSCLC patients within the same stage indicates that other factors, such as age, gender, histology, cell differentiation and the number of examined lymph nodes also contribute to the prognosis of NSCLC (4).

Recently, nomogram has been widely used to quantify risk in several types of cancers (5-7), which creates an intuitive graph of a statistical predictive model by incorporating biological and clinical variables to generate a numerical probability of a clinical event (8). Some studies have reported that nomograms are more reliable in predicting the recurrence than the traditional staging systems for patients with early stage resected NSCLC (9-12). However, few study has referred to nomograms that predict overall survival after surgery treatment in early stage NSCLC patients, particularly in Chinese population.

Thus, we aimed to construct a prognostic nomogram with demographic and clinicopathologic variables to investigate whether such nomogram would provide accurate prediction for overall survival of early stage NSCLC patients after surgery resection.

\section{Methods}

\section{Patients}

Our study was approved by the Institutional Review Board of Nanjing Medical University (FWA00001501). All patients were consecutively recruited between September 2002 and December 2011 from the Cancer Hospital of Jiangsu Province and the First Affiliated Hospital of Nanjing Medical University, Nanjing, China. All cases underwent complete resection and confirmed histopathologically by at least two qualified pathologists. TNM stage was classified according to the Union for International Cancer Control/ American Joint Committee on Cancer (UICC/AJCC) 7th stage system (3). Only patients diagnosed with pathologic stage I or pathologic stage II NSCLC were included. Those who had (I) a prior history of malignancy, (II) local advanced or metastatic disease (pathologic stage III or pathologic stage IV), (III) neoadjuvant chemotherapy and radiotherapy and (IV) a wedge resection were excluded.

The subjects were recruited after being diagnosed with lung cancer by the hospital's pathological report system. After signing informed consent, all participants were interviewed face-to-face by trained interviewers to collect demographics data (e.g., age and sex) and exposure information (e.g., smoking history and family history of cancer). Clinicopathologic variables (type of surgical resection, tumor location, station of examined lymph nodes, histology type, lymph node invasion, tumor differentiation, and pathologic TNM stage) were prospectively retrieved from the medical records.

\section{Follow-up}

All patients were followed up every 3 months through telephone contacts by trained investigators from the time of enrollment until death or last contact (last follow-up: July 2015). The follow-up data included image examination, treatment information (chemotherapy or radiotherapy) and survival status (alive, dead, time of death and cause of death). Additionally, the latest medical records were also checked as a complement. Finally, a total of 443 patients who had both follow-up and available clinical information were included in this study. Overall survival time was defined as the interval between diagnosis and the date of death or latest follow-up.

\section{Construction of the nomogram}

Continuous variables were transformed into categorical variables according to the median, such as age and station of sampled lymph nodes. In the univariate analysis, logrank test was used to compare the survival time for categorical variables and survival curves were depicted using the Kaplan-Meier method. Next, variables with $\mathrm{P}$ value $<0.05$ through above analysis entered into multivariate Cox regression model to identify independent prognostic factors. A nomogram was then formulated based on these independent prognostic factors $(\mathrm{P}<0.05)$ in the final model and delineated by using the $\mathrm{R}$ package 'rms'. The selection of the final model was using a backward step-down process 
with the Akaike information criterion (13).

\section{Validation of the NOMOGRAM}

The performance of the nomogram was assessed by Harrell's concordance index (C-index) that ranged from 0.5 (no discriminative ability) to 1 (perfect discrimination) (14). Bootstrap resamples (1,000 times) was used for internal validation of predictive accuracy and to provide a relatively unbiased estimate of C-index (13). Then, calibration plots for 3-, 5-year overall survival probability were depicted to compare the predicted survival and observed survival after the bias correction of bootstrap resample (1,000 times).

Statistical analyses were carried out using $\mathrm{R}$ software (version 3.6.1; The R Foundation for Statistical Computing, http://www.cran.r-project.org/). All tests were two-sided, with the significance level at $\mathrm{P}<0.05$.

\section{Results}

By July 2015 (the maximum follow-up time: 10.9 years; the median follow-up time: 3.5 years), 140 patients died from NSCLC and the median survival time (MST) was 8.5 years. Among 443 patients, there were 307 males $(69.30 \%)$ and 136 females $(30.70 \%)$, and the mean age was 62 years (range, 25-83 years; standard deviation, 9 years). Most of the patients had a smoking history $(58.69 \%)$, but few of them had a family history of cancer (19.64\%). The majority of NSCLC patients had adenocarcinoma (58.47\%) and squamous cell carcinoma $(36.79 \%)$, and the others included large cell and mixed-cell carcinoma (4.74\%). Detailed demographic and clinicopathologic characteristics are summarized in Table 1 .

The results of the univariate analysis indicated that patients with poor differentiation (vs. well/moderate differentiation) and less than 5 stations of sampled lymph nodes ( $v s$. more than 5 stations of sampled lymph nodes) had worse prognosis (log-rank $\mathrm{P}<0.05)$. Additionally, pathologic $\mathrm{T}$, pathologic $\mathrm{N}$ and pathologic TNM stage were also significantly associated with survival time of early stage NSCLC patients $(\log$-rank $\mathrm{P}<0.05)$ (Table 1). Then, multivariate Cox regression analysis further revealed that differentiation, station of sampled lymph nodes, pathologic $\mathrm{T}$ and pathologic $\mathrm{N}$ remained as independent prognostic factors for overall survival (Table 2). The Kaplan-Meier curves of four independent risk factors were shown in Figure 1.

Moreover, a nomogram incorporating above four independent prognostic factors was constructed to predict the 3- and 5-year overall survival for early stage NSCLC patients (Figure 2). The pathologic $\mathrm{T}$ shared the largest weight in the nomogram, followed by station of sampled lymph nodes, differentiation, and pathologic N. The calibration plot demonstrated that the predicted 3- and 5 -year overall survival probabilities corresponded closely with the actually observed probabilities (Figure 3). Finally, for predictive accuracy of overall survival, nomogram had a bia-corrected C-index of 0.622 (95\% CI: 0.572-0.672). And for the conventional TNM staging system, the C-index was 0.596 (95\% CI: 0.551-0.641).

\section{Discussion}

The TNM staging system is widely used for predicting prognosis of cancer, however, the ability of such staging system in predicting the survival of cancer was limited. In this study, we built a nomogram that well predicted the overall survival of early stage NSCLC patients after surgery resection with four independent prognostic factors (tumor differentiation, station of sampled lymph nodes, pathologic $\mathrm{T}$ and pathologic N). Furthermore, the discrimination of nomogram was comparatively stable with a fairly high C-index and the calibration plot also showed moderate goodness of fit.

In our study, the 5 -year survival in pathologic stage Ia NSCLC patients who underwent complete surgical resection was $72 \%$, which was consistent with previous study result (3). However, the 5 -year survival in stage $\mathrm{Ib}-\mathrm{IIb}$ were relatively higher than that of previous study (Ib: 66\% vs. $58 \%$; IIa: $54 \%$ vs. $46 \%$; IIb: $48 \%$ vs. $36 \%$ ) (3). Although the follow-up in our study was relatively long and regular, the limited sample size might contribute to such difference.

According to the present TNM staging system, the dominant factors for prognosis of early stage NSCLC were pathologic $\mathrm{T}(\mathrm{T} 1 \mathrm{a} / \mathrm{T} 1 \mathrm{~b} / \mathrm{T} 2 \mathrm{a} / \mathrm{T} 2 \mathrm{~b} / \mathrm{T} 3)$ and pathologic $\mathrm{N}$ (N0/N1) (3). However, for our overall survival model, the $\mathrm{C}$-index of the stage alone accounted for only approximately $60 \%$ of information, with the other factors we considered accounting for the rest (15). Our study revealed that there were other prognostic factors, including tumor differentiation and station of sampled lymph nodes, which significantly affected the overall survival of early stage NSCLC. Therefore, we added these prognostic factors in the nomogram model, and found the C-index of the model increased to 0.62 .

Prior studies were controversial about the prognostic value of tumor differentiation. For example, several studies 
Table 1 Patient characteristics and univariate analysis of predictors for overall survival of early-stage NSCLC

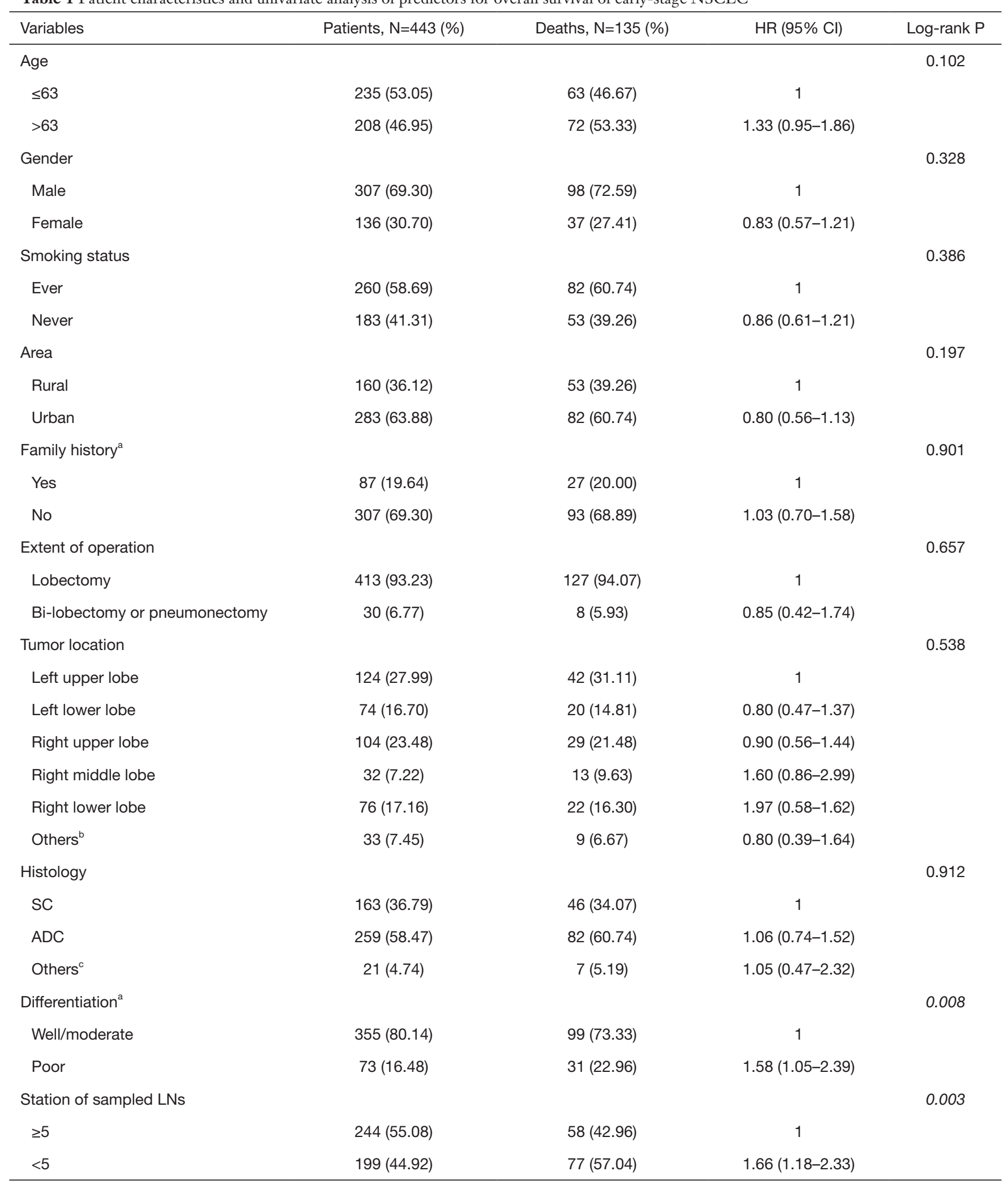

Table 1 (continued) 
Table 1 (continued)

\begin{tabular}{|c|c|c|c|c|}
\hline Variables & Patients, $\mathrm{N}=443(\%)$ & Deaths, N=135 (\%) & $\mathrm{HR}(95 \% \mathrm{Cl})$ & Log-rank P \\
\hline T1a & 68 (15.35) & $13(9.63)$ & 1 & \\
\hline $\mathrm{T} 1 \mathrm{~b}$ & 58 (13.09) & $12(8.88)$ & $1.13(0.51-2.47)$ & \\
\hline $\mathrm{T} 2 \mathrm{a}$ & $224(50.56)$ & $69(51.11)$ & $1.71(0.95-3.09)$ & \\
\hline T3 & $27(6.09)$ & $12(8.88)$ & $2.49(1.13-5.48)$ & \\
\hline Pathologic N stage & & & & 0.042 \\
\hline No & 349 (78.78) & $99(73.33)$ & 1 & \\
\hline N1 & $94(21.22)$ & $36(26.67)$ & $1.48(1.01-2.17)$ & \\
\hline IB & $186(41.99)$ & $54(40.00)$ & $1.60(0.94-2.72)$ & \\
\hline IIA & $106(23.93)$ & $37(27.41)$ & $2.29(1.30-4.01)$ & \\
\hline IIB & 54 (12.19) & $26(19.26)$ & 2.69 (1.47-4.92) & \\
\hline Treatment after surgery & & & & 0.816 \\
\hline No & $227(51.24)$ & $69(51.11)$ & 1 & \\
\hline Chemo only & $200(45.15)$ & $62(45.93)$ & $0.88(0.62-1.24)$ & \\
\hline Chemo + Rad & $16(3.61)$ & $4(2.96)$ & $0.99(0.37-2.77)$ & \\
\hline
\end{tabular}

${ }^{a}$, variables with missing data; ${ }^{b}$, others means the tumor was located at the junction between the upper left lobe and the lower left lobe, or the junction between the upper right lobe, the middle right lobe and the lower right lobe; ${ }^{c}$, others histological types include large cell lung cancer and mixed cell lung cancer. Italic $P$ values were less than 0.05 . NSCLC, non-small cell lung cancer; SC, squamous cell carcinoma; ADC, adenocarcinoma; LN, lymph node; Chemo, chemotherapy; Rad, radiotherapy.

Table 2 Independent predictors of overall survival in patients with early-stage NSCLC

\begin{tabular}{lcc}
\hline \multirow{2}{*}{ Variables } & \multicolumn{2}{c}{ Multivariable analysis } \\
\cline { 2 - 3 } Differentiation & Adjusted HR $(95 \% \mathrm{Cl})^{\mathrm{a}}$ & $\mathrm{P}^{\mathrm{a}}$ \\
Well/moderate & 1 & \\
Poor & $1.51(1.00-2.26)$ & 0.019 \\
Station of sampled LNs & 1 & \\
$\geq 5$ & $1.74(1.23-2.48)$ & 0.002 \\
$<5$ & & \\
Pathologic T stage & 1 & 0.937 \\
T1a & $1.04(0.46-2.37)$ \\
T1b & & \\
\hline
\end{tabular}

Table 2 (continued)

\begin{tabular}{lcc}
\hline \multirow{2}{*}{ Variables } & \multicolumn{2}{c}{ Multivariable analysis } \\
\cline { 2 - 3 } & Adjusted HR $(95 \% \mathrm{Cl})^{\mathrm{a}}$ & $\mathrm{P}^{\mathrm{a}}$ \\
\hline T2a & $2.87(1.01-3.46)$ & 0.046 \\
T2b & $2.85(1.17,4.79)$ & 0.020 \\
T3 & & 0.012 \\
Pathologic N stage & 1 & \\
N0 & $1.55(1.02-2.35)$ & 0.034 \\
N1 & \\
\hline a , adjusted for differentiation, stations of sampled LNs, pathologic \\
T stage and pathologic N stage where appropriate in Cox \\
regression model. Italic P values were less than 0.05. NSCLC, \\
non-small cell lung cancer; LN, lymph node.
\end{tabular}

Table 2 (continued) 

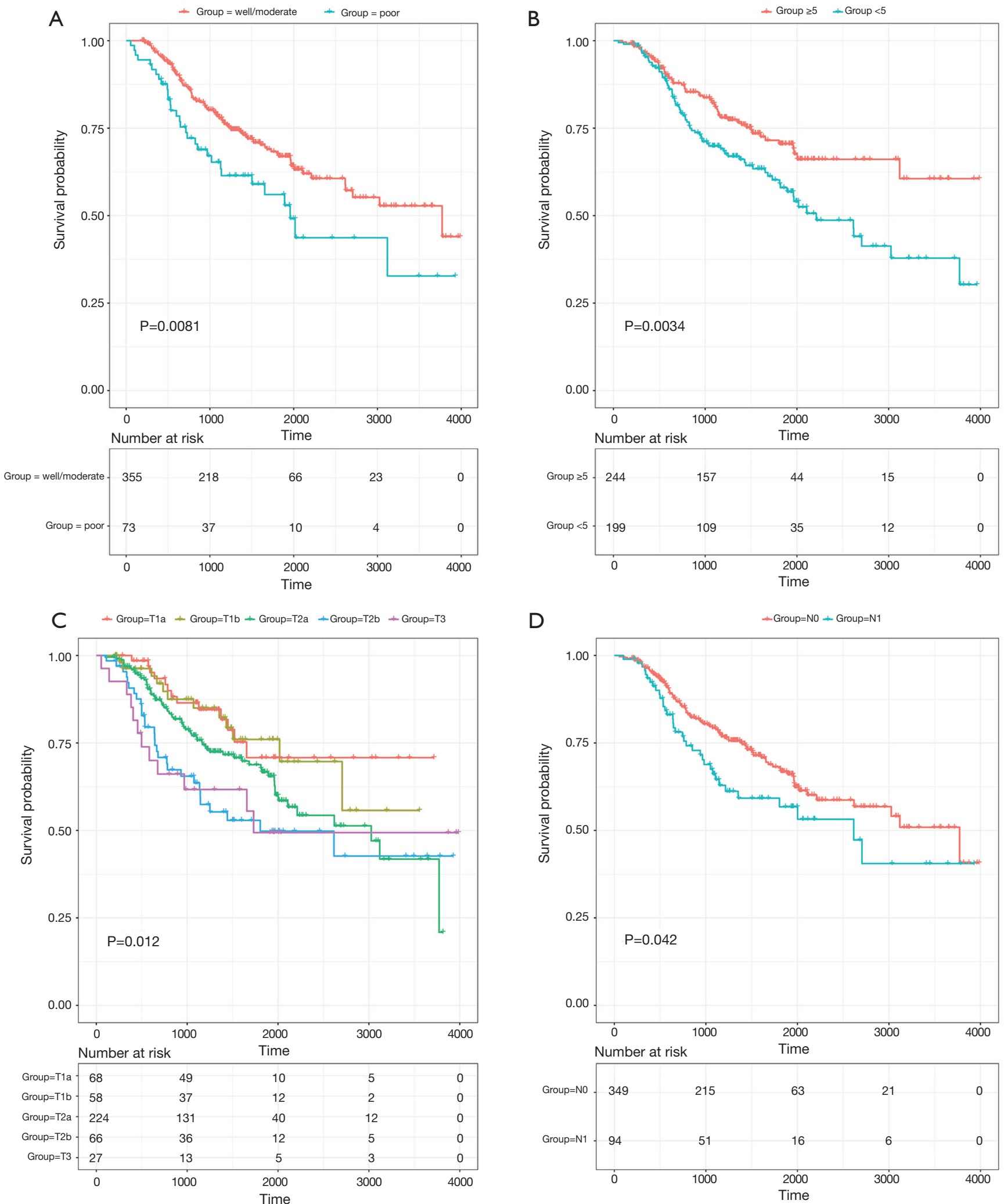

Figure 1 Kaplan-Meier curves of overall survival according to tumor differentiation (A), station of sampled lymph nodes (B), pathologic T (C) and pathologic $\mathrm{N}(\mathrm{D})$. 


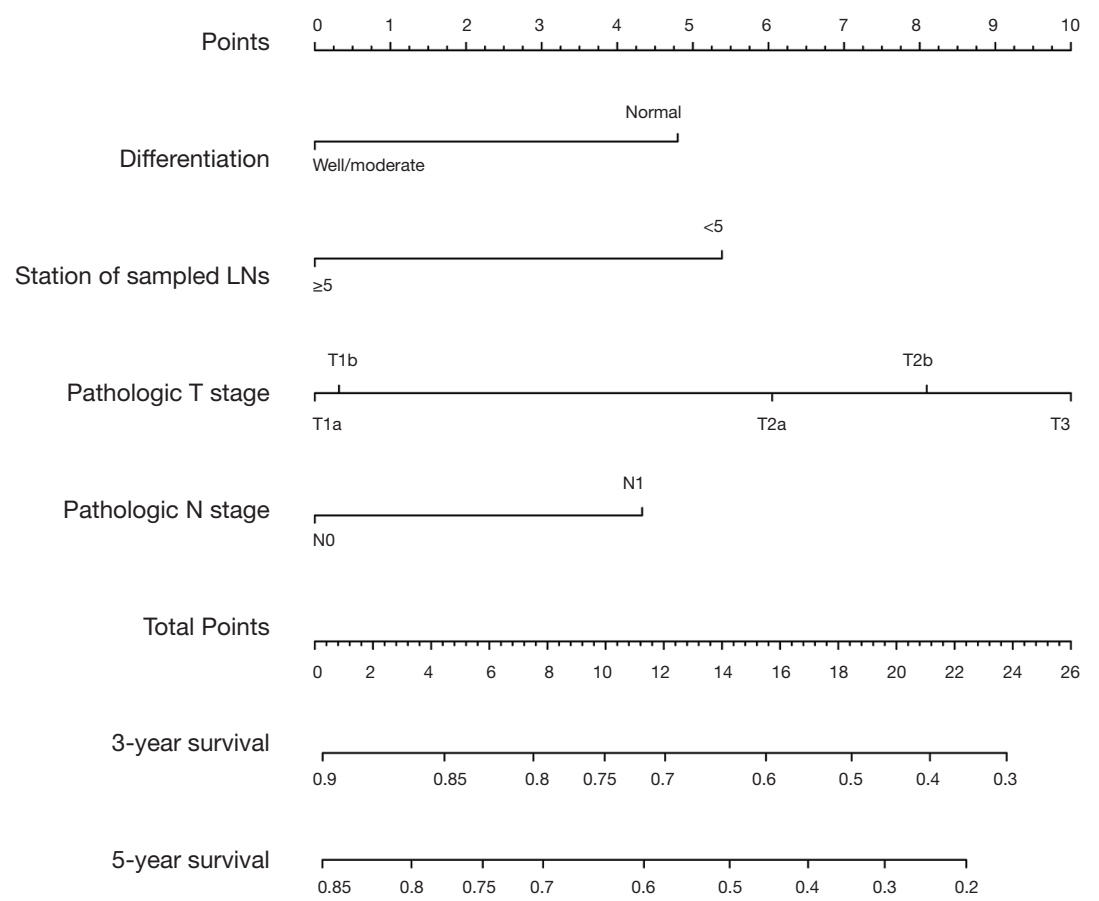

Figure 2 Postoperative prognostic nomogram for patients with early stage non-small cell lung cancer after surgery resection. To use, for example, the user should find one patient's differentiation on the differentiation axis, then draw a straight line upward to the point axis to determine how many points toward death the patient receives for differentiation. Do above process again for the other axes (station of sampled lymph nodes, pathologic $\mathrm{T}$ stage and pathologic $\mathrm{N}$ stage), each time drawing a straight line upward toward point axis. Sum points received for each predictor, and find sum on the total point axis. Draw straight line down to survival-probability axes to find patient's 3- or 5-year survival probability. LNs, lymph nodes.

A

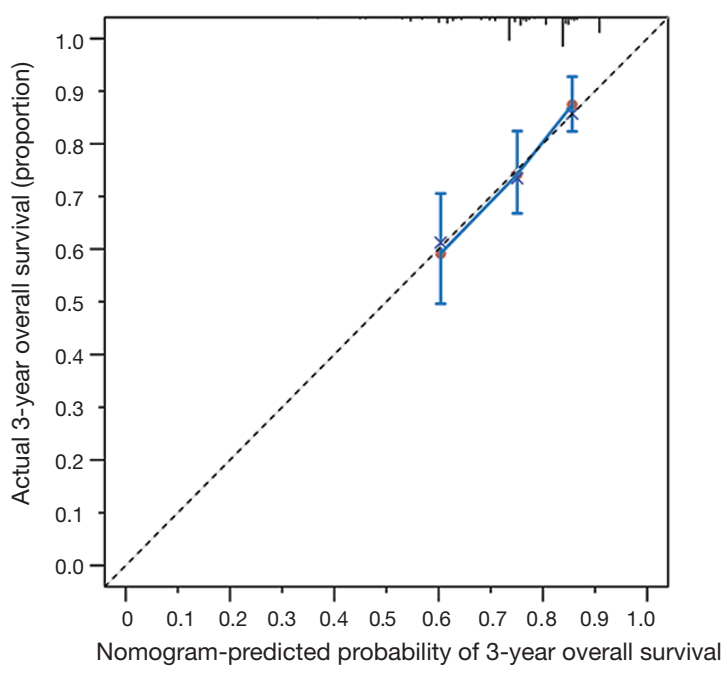

B

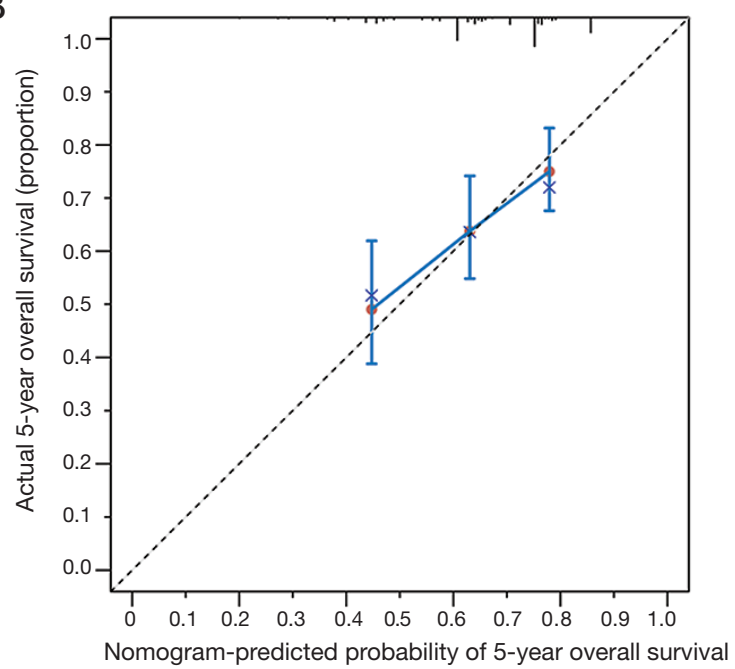

Figure 3 The calibration curves for predicting overall survival at 3-year (A) and 5-year (B). Nomogram-predicted probability of overall survival is plotted on the $\mathrm{x}$-axis; actual overall survival is plotted on the $\mathrm{y}$-axis. The solid line along the dashed line (45-degree line) would indicate a perfect calibration model in which the predicted probabilities are close to the actual probabilities. 
indicated that poorly differentiated NSCLC after resection contributed to the poor prognosis $(16,17)$; however, other studies showed that poor differentiation was not associated with the worse outcome of early stage NSCLC (18). In our study, poor differentiation was significantly associated with poor survival of early stage NSCLC, suggesting that this factor may provide useful information in defining the aggressiveness of tumor. The diversity of results might be due to sample size and non-standardized histologic grade classification of lung cancer $(16,19)$.

Previous studies mainly reported that the higher number of obtained lymph nodes was associated with the better prognosis of NSCLC $(20,21)$. The possible reason was that the thoroughness of lymph nodes sampling would determine the likelihood of clearing out the metastasized lymph nodes, and result in precise staging and therefore, guide adjuvant treatments for patients (21). However, the number of obtained lymph nodes might be confounded by fragmentation or confluence of lymph nodes (22), leading to overestimation or underestimation of accurate number of lymph nodes $(23,24)$. Thus, we used the station of sampled lymph node as a surrogate $(25,26)$. Finally, our study identified the station of sampled lymph nodes as an independent factor for overall survival in early stage resected NSCLC patients.

Nomogram, which integrates multiple risk factors to predict survival, has been identified as a useful and convenient tool in lung cancer study. For example, nomograms have been established to predict brain metastases after curative resection in NSCLC patients, the overall survival after resection of synchronous lung cancer in more than one lobe, the overall survival of stage III NSCLC patients, the response to tyrosine kinase inhibitors in EGFR-mutation NSCLC patients and the overall survival in small cell lung cancer patients (12,27-30). Whereas, for the nomogram of early stage (pathologic stage I or pathologic stage II) NSCLC, recent study mainly focused on predicting the disease-free survival after surgical resection (9,31). Sun et al. constructed a nomogram to predict prognosis after surgery in early stage nonsmall cell lung cancer in elderly patients ( $\geq 65$ years) (32). They included sex, age, pathology, differentiation, tumor diameter, and surgery type these six factors. The C-index of their study was similar to ours here, but their conclusion was for the elderly patients. Kent et al. (33) reported that age, baseline percent diffusing capacity of lung for carbon monoxide, and maximum tumor diameter were significantly associated with the survival. However, they only included clinical stage I NSCLC patients who underwent sublobar resection with or without brachytherapy, as opposed to our study that enrolled pathologic stage I and pathologic stage II patients with multiple types of surgical resection. Additionally, they did not consider the result of tumor differentiation as a potential prognostic factor.

Our study had some limitations that should be addressed. Firstly, because of missing values for some variables, we failed to integrate other potential prognostic factors, such as vascular invasion, perineural invasion, performance status, pulmonary function testing and genetic factors (e.g., EGFR mutation, ALK-EML4 fusion) (33-35). In addition, a relative small sample in this study limited the power of the statistics analysis, and there was no statistical significance in the comparison of the C-index of 7th TNM staging with our nomogram. Finally, although the method of bootstrap resampling was used to avoid overfitting (36), both predictive accuracy of the nomogram and its widespread applicability should be validated in other studies. So, further efforts on multi-center study and prospective data collection including other potential factors are encouraged to refine this nomogram.

\section{Conclusions}

We developed and validated a nomogram incorporating available clinicopathological markers to predict survival in resected early stage NSCLC patients and it showed better prediction value of survival than standard TNM staging. Such nomogram may guide appropriately postoperative decisions including intensified treatment and rigorous surveillance.

\section{Acknowledgments}

The authors wish to thank all the study participants, research staff and students who participated in this work. Funding: This work was supported by National Natural Science of China (81521004), Science Foundation for Distinguished Young Scholars in Jiangsu (BK20160046), Natural Science Foundation of Jiangsu Provience (BK20180675), Natural Science Foundation of the Jiangsu Higher Education Institutions of China (18KJB330002), a project funded by the Priority Academic Program Development of Jiangsu Higher Education Institutions (Public Health and Preventive Medicine) and Top-notch 
Academic Programs Project of Jiangsu Higher Education Institutions (PPZY2015A067).

\section{Footnote}

Conflicts of Interest: The authors have no conflicts of interest to declare.

Ethical Statement: The authors are accountable for all aspects of the work in ensuring that questions related to the accuracy or integrity of any part of the work are appropriately investigated and resolved. We further confirm that any aspect of the work covered in this manuscript that has involved human patients has been conducted with the ethical approval of all relevant bodies and that such approvals are acknowledged within the manuscript. Our study was approved by the Institutional Review Board of Nanjing Medical University (FWA00001501).

\section{References}

1. Jemal A, Bray F, Center MM, et al. Global cancer statistics. CA Cancer J Clin 2011;61:69-90.

2. Bender E. Epidemiology: The dominant malignancy. Nature 2014;513:S2-3.

3. Detterbeck FC, Boffa DJ, Tanoue LT. The new lung cancer staging system. Chest 2009;136:260-71.

4. Kawaguchi T, Takada M, Kubo A, et al. Performance status and smoking status are independent favorable prognostic factors for survival in non-small cell lung cancer: a comprehensive analysis of 26,957 patients with NSCLC. J Thorac Oncol 2010;5:620-30.

5. Han DS, Suh YS, Kong SH, et al. Nomogram predicting long-term survival after $\mathrm{d} 2$ gastrectomy for gastric cancer. J Clin Oncol 2012;30:3834-40.

6. Karakiewicz PI, Briganti A, Chun FK, et al. Multiinstitutional validation of a new renal cancer-specific survival nomogram. J Clin Oncol 2007;25:1316-22.

7. Valentini V, van Stiphout RG, Lammering G, et al. Nomograms for predicting local recurrence, distant metastases, and overall survival for patients with locally advanced rectal cancer on the basis of European randomized clinical trials. J Clin Oncol 2011;29:3163-72.

8. Balachandran VP, Gonen M, Smith JJ, et al. Nomograms in oncology: more than meets the eye. Lancet Oncol 2015;16:e173-80.

9. Zhang Y, Sun Y, Xiang J, et al. A clinicopathologic prediction model for postoperative recurrence in stage
Ia non-small cell lung cancer. J Thorac Cardiovasc Surg 2014;148:1193-9.

10. Yang HC, Kim HR, Jheon S, et al. Recurrence RiskScoring Model for Stage I Adenocarcinoma of the Lung. Ann Surg Oncol 2015;22:4089-97.

11. Zhang F, Zheng W, Ying L, et al. A nomogram to predict brain metastases of resected non-small cell lung cancer patients. Ann Surg Oncol 2016;23:3033-9.

12. Won YW, Joo J, Yun T, et al. A nomogram to predict brain metastasis as the first relapse in curatively resected nonsmall cell lung cancer patients. Lung Cancer 2015;88:201-7.

13. Harrell FE Jr, Lee KL, Mark DB. Multivariable prognostic models: issues in developing models, evaluating assumptions and adequacy, and measuring and reducing errors. Stat Med 1996;15:361-87.

14. Harrell FE Jr, Califf RM, Pryor DB, et al. Evaluating the yield of medical tests, JAMA 1982;247:2543-6.

15. Rose PG, Java J, Whitney CW, et al. Nomograms Predicting Progression-Free Survival, Overall Survival, and Pelvic Recurrence in Locally Advanced Cervical Cancer Developed From an Analysis of Identifiable Prognostic Factors in Patients From NRG Oncology/ Gynecologic Oncology Group Randomized Trials of Chemoradiotherapy. J Clin Oncol 2015;33:2136-42.

16. Sun Z, Aubry MC, Deschamps C, et al. Histologic grade is an independent prognostic factor for survival in nonsmall cell lung cancer: an analysis of 5018 hospital- and 712 population-based cases. J Thorac Cardiovasc Surg 2006;131:1014-20.

17. Shimada Y, Saji H, Yoshida K, et al. Pathological vascular invasion and tumor differentiation predict cancer recurrence in stage IA non-small-cell lung cancer after complete surgical resection. J Thorac Oncol 2012;7:1263-70.

18. Agarwal M, Brahmanday G, Chmielewski GW, et al. Age, tumor size, type of surgery, and gender predict survival in early stage (stage I and II) non-small cell lung cancer after surgical resection. Lung Cancer 2010;68:398-402.

19. Kobayashi N, Toyooka S, Soh J, et al. Risk factors for recurrence and unfavorable prognosis in patients with stage I non-small cell lung cancer and a tumor diameter of $20 \mathrm{~mm}$ or less. J Thorac Oncol 2007;2:808-12.

20. Liang W, Zhang L, Jiang G, et al. Development and validation of a nomogram for predicting survival in patients with resected non-small-cell lung cancer. J Clin Oncol 2015;33:861-9.

21. Osarogiagbon RU, Ogbata O, Yu X. Number of lymph nodes associated with maximal reduction of long-term 
mortality risk in pathologic node-negative non-small cell lung cancer. Ann Thorac Surg 2014;97:385-93.

22. Wu YC, Lin CF, Hsu WH, et al. Long-term results of pathological stage I non-small cell lung cancer: validation of using the number of totally removed lymph nodes as a staging control. Eur J Cardiothorac Surg 2003;24:994-1001.

23. Ludwig MS, Goodman M, Miller DL, et al. Postoperative survival and the number of lymph nodes sampled during resection of node-negative non-small cell lung cancer. Chest 2005;128:1545-50.

24. Bria E, Milella M, Sperduti I, et al. A novel clinical prognostic score incorporating the number of resected lymph-nodes to predict recurrence and survival in nonsmall-cell lung cancer. Lung Cancer 2009;66:365-71.

25. Doddoli C, Aragon A, Barlesi F, et al. Does the extent of lymph node dissection influence outcome in patients with stage I non-small-cell lung cancer? Eur J Cardiothorac Surg 2005;27:680-5.

26. Gajra A, Newman N, Gamble GP, et al. Effect of number of lymph nodes sampled on outcome in patients with stage I non-small-cell lung cancer. J Clin Oncol 2003;21:1029-34.

27. Oberije C, De Ruysscher D, Houben R, et al. A Validated Prediction Model for Overall Survival From Stage III Non-Small Cell Lung Cancer: Toward Survival Prediction for Individual Patients. Int J Radiat Oncol Biol Phys 2015;92:935-44.

28. Keam B, Kim DW, Park JH, et al. Nomogram Predicting Clinical Outcomes in Non-small Cell Lung Cancer Patients Treated with Epidermal Growth Factor Receptor Tyrosine Kinase Inhibitors. Cancer Res Treat

Cite this article as: Zhang J, Fan J, Yin R, Geng L, Zhu M, Shen W, Wang Y, Cheng Y, Li Z, Dai J, Jin G, Hu Z, Ma H, Xu L, Shen H. A nomogram to predict overall survival of patients with early stage non-small cell lung cancer. J Thorac Dis 2019;11(12):5407-5416. doi: 10.21037/jtd.2019.11.53
2014;46:323-30.

29. Xie D, Marks R, Zhang M, et al. Nomograms predict overall survival for patients with small-cell lung cancer incorporating pretreatment peripheral blood markers. J Thorac Oncol 2015;10:1213-20.

30. Tanvetyanon T, Finley DJ, Fabian T, et al. Prognostic nomogram to predict survival after surgery for synchronous multiple lung cancers in multiple lobes. J Thorac Oncol 2015;10:338-45.

31. Huang Y, Liu Z, He L, et al. Radiomics signature: a potential biomarker for the prediction of disease-free survival in early-stage (I or II) non-small cell lung cancer. Radiology 2016;281:947-57.

32. Sun F, Ma K, Yang X, et al. A nomogram to predict prognosis after surgery in early stage non-small cell lung cancer in elderly patients. Int J Surg 2017;42:11-6.

33. Kent MS, Mandrekar SJ, Landreneau R, et al. A Nomogram to Predict Recurrence and Survival of HighRisk Patients Undergoing Sublobar Resection for Lung Cancer: An Analysis of a Multicenter Prospective Study (ACOSOG Z4032). Ann Thorac Surg 2016;102:239-46.

34. Matsumura $Y$, Hishida T, Shimada $Y$, et al. Impact of extratumoral lymphatic permeation on postoperative survival of non-small-cell lung cancer patients. J Thorac Oncol 2014;9:337-44.

35. Yilmaz A, Duyar SS, Cakir E, et al. Clinical impact of visceral pleural, lymphovascular and perineural invasion in completely resected non-small cell lung cancer. Eur J Cardiothorac Surg 2011;40:664-70.

36. Iasonos A, Schrag D, Raj GV, et al. How to build and interpret a nomogram for cancer prognosis. J Clin Oncol 2008;26:1364-70. 